\title{
Systemic Approaches for the Potential Mechanisms of Common Respiratory Diseases Caused by Air Pollution in China
}

\section{Min Zhang ${ }^{1}$, Zhiye Fang ${ }^{1}$, Yun Zhang ${ }^{2}$, Zhigang Liu ${ }^{3}$ and Youming Zhang ${ }^{4 *}$}

${ }^{1}$ Department of Thorax Medicine, The First Affiliated Hospital of Shenzhen University, Shenzhen, PR China

${ }^{2}$ Faculty of Health and Life Sciences, Oxford Brookes University, Oxford, UK

${ }^{3}$ Institute of Allergy and Immunology, Shenzhen University, Shenzhen, PR China

${ }^{4}$ Molecular Genetics Group, National heart and Lung Institute, Imperial College London, London, UK

The air pollution due to rising in population, industrial activities, and number of vehicles in cities causes one of the major public health problems in China. A World Bank report estimated that diseases triggered by indoor and outdoor air pollution kill 750000 Chinese citizens per year [1]. Airborne particulate matter with an aerodynamic diameter less than or equal to $2.5 \mu \mathrm{m}\left(\mathrm{PM}_{25}\right)$ has become the fourth leading cause of death in China [2]. $\mathrm{PM}_{2.5}$ is able absorb toxic pollutions such as volatile organic compounds (VOCs), heavy metals, and a few polycyclic aromatic hydrocarbons (PAHs) [3]. It can penetrate deeply into the human respiratory system and reach the blood-air barrier [4]. Long-term exposure to $\mathrm{PM}_{25}$ increases the risk of respiratory diseases such as chronic cough, asthma, chronic obstructive pulmonary disease (COPD) and lung cancers. The exact mechanisms for causing respiratory diseases by air pollution components in China remain unclear.

\section{Common Respiratory Diseases and Air Pollution Components in China}

Common respiratory diseases such as chronic cough, asthma, COPD and lung cancers are complicated diseases. Genetic and environmental factors contribute to the prevalence of the diseases. Genetic and genomic studies of respiratory diseases have brought fruitful results, but only a small component of the overall genetic contribution to the diseases has been so far identified [5]. This might be because of multiple small effects, rare highly penetrant mutations or epigenetic effects. Environmental factors are the other important courses; among them air pollution is one of the most significantly prominent factors.

Recent years there was much work on the epidemiology of the air pollution related diseases in China. Epidemiological evidence indicated that the health effects of $\mathrm{PM}_{2.5}$ correlate with factors such as the population, the location, and the sources of air pollution [6]. It indicated that different locations exposed to the same level of $\mathrm{PM}_{25}$ experience different degree of toxicity due to different mechanisms. $\mathrm{PM}_{2.5}$-bound PAHs sampled from the atmosphere were found a typical southeastern China city (Guangzhou). The annual average concentration of $\mathrm{PM}_{2.5}$ was 64.88 mugm $^{-3}$. The annual average concentration of PAHs in $\mathrm{PM}_{2.5}$ was $33.89 \mathrm{ngm}^{-3}$. The significant meteorological parameters for most of the PAHs were sunshine time, air pressure, and humidity, together representing $10.7-52.4 \%$ of the variance in atmospheric $\mathrm{PAH}$ concentrations. Motor-vehicle exhaust and coal combustion were probably the main sources of PAHs in $\mathrm{PM}_{2.5}$ in Guangzhou [7].

Chronic cough is a very common complaint in clinics throughout China. Clinical and basic science research on chronic cough just began, the etiological diagnosis, treatment and pathogenesis are still in an infant stage [8]. A study found a 3.3\% incidence of chronic cough among college students in Guangzhou [9]. It is also estimated that patients who seek medical attention for chronic cough account for $30-40 \%$ of the visits to respiratory clinics in China. PM particles have been long regarded one of the causes of the chronic cough, but the relationship has not been studied fully understood and many questions remain to answer [10].
Asthma and COPD are the major respiratory complicate diseases in China. Asthma is a disease of unknown aetiology which is characterized by intermittent inflammation of the small airways of the lung with symptoms of wheeze and shortness of breath. The presence of inflammation may in time lead to irreversible airway scarring and intractable airflow limitation. Asthma cases have risen dramatically in China over the past decade along with ever deteriorating air pollution. The prevalence of asthma among urban children in China rose $64 \%$ between 1990 and 2000, approached 2\%. In bigger cities, where the air quality was lower and awareness of the ailment was higher, these figure more than doubled [11]. Chronic obstructive pulmonary disease (COPD) is a preventable and treatable disease state characterized by airflow limitation that is not fully reversible. It is associated with abnormal inflammatory response of the lungs to noxious particles or gases, primarily caused by cigarette smoking [12]. COPD is increasingly becoming a cause of public health concern and ranks first among the causes of disability. The overall prevalence rate of COPD in China is $8.2 \%$ and mortality rate because of the disease is approximately $1.6 \%$. In urban areas it is considered to be the third leading cause of death, with a prevalence rate of $6.7 \%$ to $8.3 \%[12,13]$. The major risk factors accounted for COPD in China are tobacco smoking, use of biomass fuels, and genetic susceptibility. Apart from this air pollution contributes toward an increased risk of COPD [13].

Environmental problems have intensified and the burden of lung cancer continues to increase in China as well. Throughout the last three decades, the mortality of lung cancer has dramatically increased, as shown in national death surveys. From 2000 to 2010, age specific incidence of lung cancer increased in most age groups. It is estimated that in 2015, the total number of new cases of lung cancer will reach 733300 [14].

It is now important to understand how the air pollution component $\mathrm{PM}_{2.5}$ causes the human respiratory diseases in China. $\mathrm{PM}_{2.5}$ can induce inflammation, innate and acquired immunity, and oxidative stress of airway. It can cause airway disorders including cough, infection, asthma and COPD. Understanding the mechanisms of lung diseases from $\mathrm{PM}_{2.5}$ will enhance efforts to protect at-risk individuals from the harmful respiratory effects of air pollutants. The currently available research platforms such as genomic, epigenetic and metagenomic

*Corresponding author: Youming Zhang, Molecular Genetics Group, National Heart and Lung Institute, Dovehouse Street, London SW3 6LY, UK, Tel: 207352 8121; E-mail: y.zhang@imperial.ac.uk

Received November 25, 2015; Accepted December 18, 2015; Published December 25, 2015

Citation: Zhang M, Fang Z, Zhang Y, Liu Z, Zhang Y (2015) Systemic Approaches for the Potential Mechanisms of Common Respiratory Diseases Caused by Air Pollution in China. Lung Dis Treat 1: 103. doi:10.4172/2472-1018.1000103

Copyright: @ 2015 Zhang M, et al. This is an open-access article distributed under the terms of the Creative Commons Attribution License, which permits unrestricted use, distribution, and reproduction in any medium, provided the original author and source are credited. 
approaches will play prominent roles in the search of the pathphysiologic mechanisms of the respiratory diseases caused by air pollution components in China.

\section{Genomic Approaches}

Human genome contains about 25000 genes [15]. Global gene expression profiling and RNA sequence can make it possible to examine the transcript abundance in the designed conditions of air pollution components in stimulated cells and tissues. Transcripts significant at a $1 \%$ false discovery rate (FDR) with are taken forwards for the identification of gene expression clusters in the different conditions. The cluster analysis can discover the expression patterns of genes; such methods are useful in their ability to represent varying degrees of similarity and more distant relationships among groups of closely related genes, with few assumptions about the nature of the data. The computed trees can be used to order genes in the original data table, so that genes or groups of genes with similar expression patterns are adjacent [16].

Gene silencing or gene knockdown is a power tool for decoding the networks and pathways that contain the gene of interest. When candidate genes that are responsible for air pollution components are identified, the next step would be study the path-physiology of the gene products in the mechanisms of airway diseases. With the gene specific siRNA transfection into the cells, the gene will dramatically decrease the expression. One of the powerful approaches for gene silencing is time series study. It applies stimulators of the cells at different time points and examines the phenotypes in silenced cells and control cells.

Gene over-expression in cells can be completed by expression vectors that are transfected in the cells. The interested gene is often linked with express tag such as green fluorescent protein (GFP) as a reporter of expression. This approach is not only very helpful to study the location of the gene expression, but also possible show the dynamic movement of the interested gene products after air pollution components' stimulation. The tagged reporter can also be used for coimmune precipitation if there is not good antibody available for the interested gene's product.

A knockout mouse is a genetically engineered mouse in which one or more genes have been made inoperative and is the one of the most use in gene targeting. The targeting process to generate a knockout line is usually time-consuming and difficult. The use of novel techniques such as the recombineering system in the manipulation of mouse genome is leading to more rapid generation of knockout or conditional knockout strains for genes of interest [17]. The technique of conditional gene expression applies site-specific DNA recombinase systems in mouse genome. The recent revolutionary development of zinc finger nuclease (ZFN) and CRISPR/Cas9 systems offer other means for genomic editing [18].

Metabolite screening can be applied in the cell level and in animal studies after stimulation by air pollution components. It not only provides new insight of the metabolism of the gene's product but also possible identifies new biomarkers for the diseases. Pharmacologic approaches include screening the inhibitors of the pathways that the genes products are involved in and to examine the phenotypes of the cells after inhibition. Global gene expression profiling can also be integrated in pharmacologic approaches for the dissection of the gene's functions. A recent transcriptomic analyses of airborne $\mathrm{PM}_{2.5}$ in Beijing exposure on human bronchial epithelial cells showed $\mathrm{PM}_{2.5}$ induced genes involved in responses to xenobtiotic stimuli, metabolic response, and inflammatory and immune response pathways such as
MAPK signaling and cytokine-cytokine receptor interaction, which might contribute to $\mathrm{PM}_{25}$-related respiratory diseases [19].

\section{Epigenetic Approaches}

Epigenetic regulation modifies gene expression that is not caused by changes in the DNA sequence but by DNA methylation, histone modification and other mechanisms. DNA methylation involves the addition of a methyl group to the DNA nucleotide cytosine and adenine which lead to gene silencing. Histones are highly alkaline proteins found in eukaryotic cell nuclei that package and order the DNA into nucleosome. The major histone modifications are methylation, acetylation, phosphorylation, ubiquitination and simulation. The effects of such modifications range from gene activation to gene silencing. Epigenetic effects are other possible causes of familial clustering. The patterns of gene expression that determine cellular types and function become stably restricted during development, partly through methylation of $\mathrm{CpG}$ sequences and gene silencing. A good example for environmental factor influencing epigenetic regulation came from the evidence from children who lived in farm. Children growing up in a traditional farming environment had lower risk of allergic respiratory diseases. Demethylation of the FOXP3 promoter was association with higher number of FOXP3 cells in cord blood mononuclear cells in an extensive farming exposure environment [20]. Recent epigenomewide approach identified 36 loci that had association of serum IgE level [21]. Air pollution components have all been strongly associated with altered methylation at selected loci. The epigenetic approaches for air pollution components will add new insight of the gene regulation in common respiratory diseases in China.

\section{Metagenomic Approaches}

Human microbiota is diverse across individuals and different sites in the body. 16S rRNA gene sequencing has been the first molecular tool to be generally applied to the human microbiota. It gives a quantitative description of the bacteria present in a complex biological mixture, allowing investigation of whole communities and the identities of their constituent members [22]. Oral microbiota are known to be involved in upper and lower respiratory infections, which can also develop into airway diseases such as bronchial asthma and COPD [23,24]. Although strong evidence exists to implicate bacterial infections in the course and pathogenesis of airway diseases, systematic studies of organisms and how to interact with air pollution components in respiratory diseases in China are lacking. Recent high throughput methods like next-generation sequencing have revolutionized the way diversity of microbial communities in varied environments. The metagenomic approaches of air pollution components can lead to identification of potential targets for respiratory diseases in China.

In summary, systemic approaches from genomic to metagenomic research on air pollution components will provide an unprecedented insight into the nature of the respiratory disease mechanisms. Combining these approaches together with molecular biologic, immunologic studies will bright new insight into the complicated respiratory diseases that are caused by air pollution components in China. It will also provide new ways to prevent and treat the diseases in the future.

\section{References}

1. Chang $Y$ (2012) China needs a tighter PM2.5 limit and a change in priorities Environ Sci Technol 46: 7069-7070.

2. Chen Z, Wang JN, Ma GX, Zhang YS (2013) China tackles the health effects of air pollution. Lancet 382: 1959-1960.

3. Cassee FR, Heroux ME, Gerlofs-Nijland ME, Kelly FJ (2013) Particulate 
Citation: Zhang M, Fang Z, Zhang Y, Liu Z, Zhang Y (2015) Systemic Approaches for the Potential Mechanisms of Common Respiratory Diseases Caused by Air Pollution in China. Lung Dis Treat 1: 103. doi:10.4172/2472-1018.1000103

matter beyond mass: recent health evidence on the role of fractions, chemical constituents and sources of emission. Inhal Toxicol 25: 802-812.

4. Dockery DW (2009) Health effects of particulate air pollution. Ann Epidemio 19: $257-263$

5. Zhang Y, Moffatt MF, Cookson WO (2012) Genetic and genomic approaches to asthma: new insights for the origins. Curr Opin Pulm Med 18: 6-13.

6. Shang Y, Sun Z, Cao J, Wang X, Zhong L, et al. (2013) Systematic review of Chinese studies of short-term exposure to air pollution and daily mortality. Environ Int 54: 100-111.

7. Liu J, Man R, Ma S, Li J, Wu Q, et al. (2015) Atmospheric levels and health risk of polycyclic aromatic hydrocarbons (PAHs) bound to PM2.5 in Guangzhou, China. Mar Pollut Bull 100: 134-143

8. Lai K1, Luo W, Zeng G, Zhong N (2012) Diagnosis and treatment of chronic cough in China: an insight into the status quo. See comment in PubMed Commons below Cough 8: 4

9. Chen RC, Lai KF, Liu CL, Luo W, Zhong NS (2006) An epidemiologic study of cough in young college students in Guangzhou. Zhonghua Liu Xing Bing Xue Za Zhi 27: 123-126.

10. Mao W, Xia W, Chen J (2013) Air pollution and chronic cough in China. Chest 144: 362-363.

11. Watts J (2006) Doctors blame air pollution for China's asthma increases. Lancet 368: 719-720.

12. Celli BR, MacNee W; ATS/ERS Task Force (2004) Standards for the diagnosis and treatment of patients with COPD: a summary of the ATS/ERS position paper. Eur Respir J 23: 932-946.

13. Gao J, Prasad N (2013) Chronic obstructive pulmonary disease in China: the potential role of indacaterol. J Thorac Dis 5: 549-558.
14. Chen W, Zheng R, Zeng $\mathrm{H}$, Zhang S (2015) Epidemiology of lung cancer in China. Thorac Cancer 6: 209-215.

15. Stein LD (2004) Human genome: end of the beginning. Nature 431: 915-916.

16. Eisen MB, Spellman PT, Brown PO, Botstein D (1998) Cluster analysis and display of genome-wide expression patterns. Proc Natl Acad Sci U S A 95: 14863-14868.

17. Liu P, Jenkins NA, Copeland NG (2003) A highly efficient recombineering based method for generating conditional knockout mutations. Genome Res 13: 476-484.

18. Urnov FD, Rebar EJ, Holmes MC, Zhang HS, Gregory PD (2010) Genome editing with engineered zinc finger nucleases. Nat Rev Genet 11: 636-646.

19. Zhou Z, Liu Y, Duan F, Qin M, Wu F, et al. (2015) Transcriptomic Analyses of the Biological Effects of Airborne PM2.5 Exposure on Human Bronchial Epithelial Cells. PLoS One 10: e0138267.

20. Schaub B, Liu J, Höppler S, Schleich I, Huehn J, et al. (2009) Maternal farm exposure modulates neonatal immune mechanisms through regulatory $\mathrm{T}$ cells. J Allergy Clin Immunol 123: 774-782.

21. Liang L, Willis-Owen SA, Laprise C, Wong KC, Davies GA, et al. (2015) An epigenome-wide association study of total serum immunoglobulin $E$ concentration. Nature 520: 670-674.

22. Cox MJ, Cookson WO, Moffatt MF (2013) Sequencing the human microbiome in health and disease. Hum Mol Genet 22: R88-94.

23. Hilty M, Burke C, Pedro H, Cardenas P, Bush A, et al. (2010) Disordered microbial communities in asthmatic airways. PLoS One 5: e8578.

24. Molyneaux PL, Mallia P, Cox MJ, Footitt J, Willis-Owen SA, et al. (2013) Outgrowth of the bacterial airway microbiome after rhinovirus exacerbation of chronic obstructive pulmonary disease. Am J Respir Crit Care Med 188: 1224 1231. 